\title{
NO WORDS FOR EMOTIONS: EMOTIONAL CREATIVITY AND ALEXITHYMIA IN ART
}

\author{
Nino Abuladze, Khatuna Martskvishvili \\ Ivane Javakhishvili Tbilisi State University, Georgia \\ Email: khatuna.martskvishvili@tsu.ge,nini_abuladze17@yahoo.com
}

\begin{abstract}
The present research investigates emotional characteristics of professional culture - artists, examining the difference between art and non-art faculty students in emotional creativity and exploring the relationship between emotional creativity and alexithymia. Emotional creativity refers to the person's ability to express and experience authentic, original and appropriate combinations of emotions. Art and non-art faculty students from different universities (34 female and 46 males; $M$ of age $=20.41, S D=1.64$ ) were administered with the Emotional Creativity Inventory (ECI) along with the 20-Item Toronto Alexithymia Scale (TAS-20) The result showed that art faculty students have higher scores on emotional creativity than students from other faculties. Emotional creativity aspects are negatively related with the alexithymia. In the interpretation of results, emotional creativity is represented as one of the psychological constructs which is an important characteristic for the individuals working in the field of art. There's an illustration how differently artistic individuals understand and experience emotions. The results have practical implication for social and emotional learning perspective. According to following research not only cognitive but emotional creativity is an important disposition for creative work. This emotional aspect should be identified at school age to contribute the development of individuals' artistic skills.
\end{abstract}

Key words: artistic skills, alexithymia, emotional creativity.

\section{Introduction}

Numerous studies have been conducted about the role of creativity in art work (Zacks, J.M. Magliano (2011), Kreitler (1972). But the focus of these studies was cognitive creativity. Although creative work is often somehow linked to emotions, we have a little specific information about the role of emotions in creative work. As cognitive creativity is the key component of cognitive processes involved in creative work of art, we suppose that emotional creativity should be an important part of creative work.

The concept of emotional creativity contains the characteristics which might be associated with professional group of artists. The studies related to emotional creativity (Averill, 2005, Gutbezahl, \& Averill, (1996) are logical contributions to our assumptions. We review the meaning of emotional creativity and related studies in order to define the nature of the concept by which we characterize artists. 
Concept of Emotional Creativity is derived from social-constructivist view of emotions. The view rests on fundamental assumptions that emotions are complex syndromes, no one component of which is essential to the whole, with social norms providing the primary though not exclusive organizing principles (Averill, 1980). Averill suggests that emotional creativity is logically derived from social-constructivist view of emotions: what societies construct, individuals can reconstruct. If the reconstruction meets the criteria of novelty, effectiveness, and authenticity, it can be considered emotionally creative (Averill, 2005).

Averill defined emotional creativity as one's ability to feel and express emotions honestly, as well as in unique ways that are effective in meeting the demands of both intra and interpersonal situations. The concept consists of four dimensions that theoretically build the concept. Empirical evidence contributed to the theoretical model. The main criterion for emotional creativity is novelty (Averill \& Thomas-Knowles, 1991). Creative emotion should be in some extent new, different or unusual (Averill \& Thomas-Knowles, 1991). But novelty isn't enough. Creative response should be distinguished from bizarre, merely eccentric, random emotion (Averill \& Thomas-Knowles, 1991). Authenticity is what distinguishes it from other types of novel response. Creative response is supposed to be authentic, genuine, expressing real self (Averill \& Thomas-Knowles, 1991). We should consider that authenticity requires that a person stands alone, against social custom, expectations of family and friend, but not always. Sometimes people are more authentic when they conform than when they diverge from social expectations (Thomas-Knowles\& Averill, 1991). To be considered creative emotional response must be of some potential benefit or value for individual or society (Averill \& Thomas-Knowles, 1991). That means emotional response should be effective for achieving the aims inherent to emotions. The authors argue that effectiveness is relative concept. What is effective in one context, may be ineffective in another context of what is effective in short term run might be detrimental in long term run and vice versa (Averill \& Thomas-Knowles,1991).

Based on the general dynamics of creative process researchers concluded that before creative emotion occurs, it requires previous stages of development: preparation, incubation, illumination and verification (Wallas, 1926). Novelty, effectiveness and authenticity are the criteria matching to the verification stage. Incubation and illumination phases are hard to observe and reveal. J. Averill (1999) included in emotional creativity theory the criteria of preparedness to identify individual's interest and extent of thought about his/her own emotions.

For better understanding the construct of emotional creativity we should know how it is related to cognitive intelligence and emotional intelligence (Ivcevic et al. 2007). J. Averill (2004) suggests that emotional creativity stands to emotional intelligence as cognitive creativity stands to cognitive intelligence. Emotional creativity requires some degree of emotional intelligence while emotional intelligent people may not be emotionally creative.

Research revealed that cognitive and emotional creativity are different constructs and they do not overlap. Researchers (Averill \& Thomas-Knowles, 1991) found relationships between 4 psychological instruments: Emotional creativity, Emotional consequences test, Cognitive creativity and Cognitive consequences test. Correlations between two instruments that measure the same domain (cognitive or emotional) are stronger than correlations between the instruments that measure the same dimensions of different domains (Averill, \&Thomas-Knowles, 1991).

\section{Characteristics of Emotionally Creative Individuals}

Based on empirical studies we assume that emotionally creative individuals are opened to experience (Averill, 1999). People who frequently experience new emotions have higher degree of neuroticism (Averill, 1999). Emotional creativity is associated to impulsiveness (Averill, 1999). It should be mentioned that emotional creativity is not linked to affect intensity (Averill. 1991). Creative emotions don't mean intense or strong emotions (Averill, 1991).

Emotional creativity is linked to traumatic experience. The author of the concept argued and empirically confirmed that traumatic experience, obstacles and frustration contributes 
OF PSYCHOLOGY IN THE $21^{\text {st }}$ CENTURY Vol. 10, No. 2, 2016

64

to developing emotional creativity (Averill, 1999). Another explanation is that emotionally creative people are able to process and interpret frustrating experience differently if we in addition consider another finding that emotionally creative people are able to enjoy the benefits of solitude (Long, Averill, 2003). Emotional creativity is correlated to the third scale of solitude inventory, which includes inner calmness, self-discovery and privacy (Long., \& Averill, 2003). One important empirically based assumption is that emotionally creative people more frequently use avoidance as dominant coping strategy (Averill, 1999). These characteristics logically might be related to artist's personality if we suggest that art work may become the avoidance strategy and those who are able to spend time with their art work enjoy benefits of solitude (Although we should consider the type of creative work).

The important observation was that emotionally creative people better express their emotions by symbols (Gutbezahl\& Averill, 1996). Their narratives and paintings are distinguished by diversity and unusual combinations of contradicted traits. It means that some people who are more comfortable at using symbols to express their ideas or emotional states have more creative emotions than others (Gutbezahl \& Averill, 1996).

\section{Alexithymia}

People with alexithymia have difficulty expressing and experiencing the most basic emotions (Bagby et.al (1994). They lack empathy and have difficulties to identify, describe their emotional states, it decreases their social skills. They are oriented more on facts than on feelings and inner experiences.

Empirical study showed relationship between Alexithymia and Emotional Creativity (Averill, 1999) Total Alexithymia and total ECI (Emotional Creativity Inventory) found to be correlated negatively. ECI subscales are negatively associated with Alexithymia subscales: externally oriented thinking. Authenticity/effectiveness showed negative correlations to difficulty identifying feelings and difficulty describing feelings, but novelty showed correlations in opposite direction: there is positive relationship between novelty subscale of ECI and two scales of Alexithymia: difficulty identifying and describing feelings. As Averill (1999) explains, "These data indicate that people have difficulties identifying and describing emotions for two very different reasons, as evidences two very different outcomes: for alexithymics the difficulty is due to vagueness and shallowness of experience; for emotionally creative people it is due to complexity and originality of experience".

\section{Present Research}

The aim of present study is to investigate if professional group of artists differ from other population by emotional creativity. We examined statistical differences between art and nonart faculty students.

According to our hypothesis, artists are more emotionally creative than non-artists. The logic follows from J. Averill's (1996) study which revealed that emotional creativity is best expressed by symbols. Another theoretical assumption is that emotions are as significantly involved in art work as cognitive processes. If creativity is the most important from cognitive domain in art process, emotional creativity might have the same importance as the dimension of emotional domain.

\section{Methodology of Research}

The quantitative research has been designed to explore the relation of emotional creativity with alexithymia in an undergraduate student sample: art and non-art faculty students $\mathrm{T}$ test was used to determine the difference between groups. A multiple regression has been used to check whether Alexithymia predicts emotional creativity. T test and linear regression were used as statistical method for data processing in SPSS. 
Emotional Creativity. Emotional Creativity was measured by Emotional Creativity Inventory (ECI) (Averill, 1999). The inventory was developed by J. Averill to measure individual differences, according to emotional creativity. Based on the theory, questionnaire consists of 4 subscales: novelty ( 14 items), effectiveness ( 5 items), authenticity ( 4 items) and preparedness (7 items). The inventory contained the items like ,the range and diversity of my emotions sometimes exceed my ability to describe them”, „I prefer movies and books that depict complex and improbable emotions”, „I try to imagine situations that call for unusual, uncommon, unconventional emotions".

Alexithymia. We used a self-report measure of alexithymia: Toronto Alexithymia scale (TAS-20; Bagby, Parker, \& Taylor, 1994). The scale consisted of 20 items (e.g. "It is difficult for me to find right words for feelings" "I prefer talking to people about their daily activities rather than their feelings"). The scale measures three factors: difficulty identifying feelings ( 7 items), difficulty describing feelings (5 items), and externally oriented thinking (8 items).

The participants were instructed to indicate their agreement with each item on 5-point Likert scale ranging from 1 (Not at all true) to 5 (Totally true). Original version of ECI was translated and adopted on Georgian sample. The reliability of ECI has been established with satisfactory internal consistency $(\alpha=.79)$. The scales of effectiveness and authenticity have not as acceptable reliability coefficients as in original version $(\alpha=.90)$. We might consider that the scales are measured by relatively few items than remained two scales, which have sufficient reliability coefficient. If we consider the sample size of present research, we can assume these results as acceptable.

Table 1. Means, standard deviations, and internal consistencies for emotional creativity and alexithymia.

\begin{tabular}{lccccc}
\hline & N & $\begin{array}{c}\text { Number of } \\
\text { Items }\end{array}$ & $\begin{array}{c}\text { Cronbach's } \\
\text { alphas }\end{array}$ & M & SD \\
\hline Emotional Creativity & 78 & 30 & .79 & 107.71 & 13.36 \\
Novelty & 78 & 14 & .74 & 24.63 & 4.76 \\
Preparedness & 79 & 7 & .71 & 52.63 & 8.33 \\
Effectiveness & 79 & 5 & .57 & 16.81 & 3.05 \\
Alexithymia & 73 & 20 & .73 & 57.56 & 8.33 \\
Difficulty identifying & 79 & 5 & .36 & 14.02 & 3.64 \\
Difficulty describing & 78 & 7 & .69 & 18.76 & 6.02 \\
Externally oriented thinking & 75 & 8 & .24 & 25.06 & 4.07 \\
\hline
\end{tabular}

The internal consistence for both scales reached optimal level for personality questionnaires (Table 1). Statistical links were examined between emotional creativity and alexithymia, which is described as inability to understand inter and intrapersonal emotions, express and regulate them. Logically, being emotionally creative requires flexibility and development in emotional domain. Empirical studies revealed negative relationship between the two concepts (Averill, 1999), (Fuchs, Kumar, \& Porter (2007). We duplicated the research for the purpose of validation ECI.

\section{Research Participants}

Participants were 80 students from Georgian Universities between the age 18 to 25 $(M=20.41, S D=1.643), 46$ men and 34 women. One group was recruited on art faculties $(40$ students), 24 men and 16 women $(M=20.7, S D=1.8)$. Another group - from non- art faculties (40 students), 18 women and 22 men, $(M=20.1, S D=1.4)$. 
OF PSYCHOLOGY IN THE $21^{\text {st }}$ CENTURY Vol. 10, No. 2, 2016

\section{Results of Research}

Statistical analysis showed significant difference between art and non-art individuals using T-test analysis. Artist $(\mathrm{M}=12.80, \mathrm{SD}=1.46)$ were more emotionally creative than nonartists $(M=11.71, \mathrm{SD}=1.56, \mathrm{t}(76)=3.167, \mathrm{p}=.002)$. There was significant difference between two groups according to novelty and effectiveness-authenticity scales. Specifically, on novelty subscale artists group scored higher $(\mathrm{M}=3.94, \mathrm{SD}=0.51)$ than non-artists $(\mathrm{M}=3.77, \mathrm{SD}=0.91, \mathrm{t}$ $(76)=2.87, \mathrm{p}=.005)$ According to effectiveness artists group scored higher $(\mathrm{M}=3.63, \mathrm{SD}=0.79)$ than non-artists $(\mathrm{M}=3.23, \mathrm{SD}=0.50, \mathrm{t}(77)=1.989, \mathrm{p}=.05)$. But there were no difference between two groups according to preparedness subscales of emotional creativity.

Relationships between ECI and Alexithymia and their subscales were also checked.

- Novelty scale was positively correlated to the first two scales of Alexithymia: Difficulty of describing emotions and $(\mathrm{r}=0.234, \mathrm{p}<0.05)$ and difficulty to identify emotions $(\mathrm{r}=0.367, \mathrm{p}<0.01)$.

- Emotional creativity was negatively associated with the third scale of alexithymia: externally oriented thinking $(\mathrm{r}=-0.437, \mathrm{p}<0.01)$.

- $\quad$ Effectiveness / authenticity scales were negatively correlated to the alexithymia and its subscales $(\mathrm{r}=-0.370, \mathrm{p}<0.01)$.

No differences between two groups of art and non-art faculty students, according to alexithymia scale were found. There were no significant gender differences.

The predictive efficacy of alexithymia for emotional creativity was examined. The results of linear multiple regression provided support for theoretical model, that alexithymia is significant predictor of emotional creativity $\left(\mathrm{F}(1,69)=9.61, \beta=.35, \mathrm{p}<.05, R^{2}=1.22\right)$. Difficulties in emotional aspects predict low scores on emotional creativity.

\section{Discussion}

The fact that emotional creativity is linked to arts is logical, because emotional creativity is best expressed by symbols (Gutbezahl \& Averill, 1996). The ability to better express inner states by symbols is linked to emotional creativity. One explanation is that individuals who are highly emotionally creative can't express their extraordinary emotions in usual situations of everyday life. They tend to find another way to express their unusual inner feeling by symbols, so they are inclined to be involved in art work.

It is possible that unusual combination of emotions inspire them to make creative masterpieces. Another interpretation is that the process of art itself causes unusual and intense emotions emotionally creative people seek for.

The nature of art work is so much complex that maybe cognitive creativity is just one part of creativity in art work and emotional creativity is another significant part of it if we consider that artist often talk about their emotions linked to their creative work. Our results do not fully explain the role of emotions in art process but we can conclude that people involved in art differ from other population by specific characteristics that are described by concept of emotional creativity. Although it should be mentioned that emotional creativity may not be the best construct fully explaining the difference. But we suppose and our results contribute to the theory that emotional creativity is good enough to discriminate emotional characteristics of art and non-art individuals.

Social constructivist view of emotion suggests that the way people express and experience emotions is defined by cultural rules (Averill, 1980). Emotionally creative people can destroy the rules and find the new way. It is concluded that in art process emotionally creative individuals are more able to feel free from cultural schemas and express inner states in non-standard way in arts.

Correlational analysis shows combined results: Emotional Creativity was not significantly correlated to Alexithymia because of negative and positive correlations of their subscales Our results of subscale correlations match to original study (Averill, 1999).Novelty scale of ECI is positively correlated to Difficulty of describing emotions We found that Novel emotions are 
also linked to difficulty of identifying emotions. The interpretation of these results is that as J. Averill (1999) argues difficulty of describing emotions is caused by two different reasons: Inability to describe feelings or the complexity and diversity of emotions. We may say the same about the difficulty to identify emotions: creative emotions are different from existing schemas to describe them and people find difficulties with categorizing emotions.

Regression analysis contributed to the hypothesis that being emotional creativity requires some degree of emotional development.

The limit of this study was that we used self-report inventory to measure emotional characteristics. Results could be more reliable if we use objective measurements. The sample size was not enough to generalize the results. It should be better if we investigated artists instead of art faculty students. Recommendation for future research is to choose the sample from professional artists. In additions future researches should find differences between different types of art.

\section{Conclusions}

Research give an insight to define the role of individual differences in emotional domain for self-actualization. People's tendency to create is driven by emotional characteristics. The concept of emotional creativity gives us further information about artistic individuals. Emotionally Creative people may expose their inner states and feelings by creative process. This is the little part of understating the personality behind arts. In future perspective we may investigate artists and more specifically conceptualize emotional traits linked to particular art process - specifically creative activity, find out the influence of emotional creativity on performance level. It is recommended to investigate how emotional creativity leads people to art by qualitative research.

Present research provides practical implication for better educational outcomes as well. If emotional creativity is a psychological trait associated to artistic nature, it's important to identify this emotional aspect at school age to contribute the development of artistic skills.

\section{References}

Averill, J. R. (1980). A constructivist view of emotion. In R. Plutchik \& H. Kellerman (Eds.), Theories of emotion (pp. 305-340). New York: Academic Press.

Averill, J. R., Thomas-Knowles, C. (1991). Emotional creativity. International review of studies on emotion (vol. 1, pp 269-299). London; Wiley.

Averill, J. R. (1999). Individual differences in emotional creativity: Structure and correlates. Journal of Personality, 67, 331-371.

Averill, J. R., Chon, K. K., \& Hahn, D. W. (2001). Emotions and creativity, East and West. Asian Journal of Social Psychology, 4, 165-183.

Averill, J. R. (2004). A tale of two sharks: Emotional intelligence and emotional creativity compared. Psychological Inquiry, 15, 228-233.

Averill, J. R. (2005). Emotions as mediators and as products of creative activity, Creativity across domains: Faces of the muse (pp. 225-243). Mahwah, NJ: Erlbaum.

Bagby, R. M., Parker, J. D. A., \& Taylor, G. J. (1994). The twenty item Toronto alexithymia scale I. Item selection and cross validation of the factor structure. Journal of Psychosomatic Research, 38 (1), 23-32.

Fuchs, G. L., Kumar, V. K., \& Porter, J. (2007). Emotional creativity, alexithymia, and styles of creativity. Creativity Research Journal, 19, 233-145.

Gutbezahl, J., Averill, J. R. (1996). Individual differences in emotional creativity as manifested in words and pictures. Creativity Research Journal, 9, 327-337.

Ivcevic, Z., Brackett, M., \& Mayer, J. (2007). Emotional intelligence and emotional creativity. Journal of Personality, 75 (2), 199-236.

Long, C. R., Averill, J. R. (2003). Solitude: An exploration of benefits of being alone. Journal for the Theory of Social Behavior, 33, 21-44. 
OF PSYCHOLOGY IN THE $21^{\text {st }}$ CENTURY

Vol. 10, No. 2, 2016

68

Wallas, G. (1926). The art of thought. New York, Harcourt, Brace and Company.

Zacks, J. M., Magliano, J. (2011). Film, narrative, and cognitive neuroscience. In D. P. Melcher and F. Bacci (Eds.), Art and the senses. Oxford: Oxford University Press.

Kreitler, H., Kreitler, S. (1972). Psychology of the arts. Durham, NC: Duke University.

Received: November 19, 2016

Accepted: December 22, 2016

Nino Abuladze $\quad$ MSC in Psychology, Psychology Department, Faculty of Psychology and Education Sciences, Tbilisi State University, Chavchavadze av.\#1, 0179 Tbilisi, Georgia.

Khatuna Martskvishvili
PhD, Associate Professor, Psychology Department, Faculty of Psychology and Education Sciences, Tbilisi State University, Chavchavadze av.\#1, 0179 Tbilisi, Georgia.

E-mail: Khatuna.martskvishvili@tsu.ge 